\title{
Crystallinity, surface morphology, and photoelectrochemical effects in conical InP and InN nanowires grown on silicon
}

Vijay Parameshwaran ${ }^{1,2}$, Xiaoqing $\mathrm{Xu}^{3}$, and Bruce Clemens ${ }^{2, *}$

${ }^{1}$ Department of Electrical Engineering, Stanford University, Stanford, California 94305, United States

2 Department of Materials Science and Engineering, Stanford University, Stanford, California 94305, United States

3 Stanford Nanofabrication Facility, Stanford University, Stanford, California 94305, United States

* Corresponding e-mail address: bmc@stanford.edu 


\section{Materials and methods}

\section{MOCVD growth of InP nanowires}

The growth substrates are single crystal (100) boron-doped silicon with a resistivity of 0.001 $0.005 \Omega$-cm (MTI Corp.) and (100) zinc-doped GaAs wafers with a nominal carrier density of $1 \times 10^{19} \mathrm{~cm}^{-3}$ (Wafer Technology Ltd.). The silicon substrates are cleaved into $1 \mathrm{~cm}$ by $1 \mathrm{~cm}$ pieces with a diamond scribe and wafer cleaving pliers and rinsed sequentially with acetone, methanol, and isopropanol to remove any powder residue on the surface. The silicon substrates are dipped in a $5 \% \mathrm{HF}$ solution to remove native $\mathrm{SiO}_{2}$. The GaAs substrates are dipped in a $5 \% \mathrm{HCl}$ solution to remove native gallium oxide.

The substrates are loaded into an Aixtron AIX 200/4 MOCVD system through a nitrogen glovebox. A bakeout step is performed for 2 minutes at $800{ }^{\circ} \mathrm{C}$ with a system pressure of 20 mbar, and then for 10 minutes at $800{ }^{\circ} \mathrm{C}$ with a system pressure of 950 mbar. The temperature is taken down to $375{ }^{\circ} \mathrm{C}$ for the growth process. Indium droplets are deposited by decomposing TMIn at $375{ }^{\circ} \mathrm{C}$ and 800 mbar for 15 seconds. InP nanowires are grown with precursor flow rates of TMIn $=12.168 \mu \mathrm{mol} / \mathrm{min}$ and $\mathrm{TBP}=348.592 \mu \mathrm{mol} / \mathrm{min}$ for 16 minutes. No active dopant source is flowed, and the system pressure is maintained at 100 mbar. Once growth is finished, the system is cooled down under a TBP overpressure. A variation of the InP nanowire synthesis has the growth step proceed for 8 minutes, after which the TMIn source is turned off and the process continues at a temperature of $375{ }^{\circ} \mathrm{C}$ under TBP flow rate of $348.592 \mu \mathrm{mol} / \mathrm{min}$ for another 8 minutes before cooldown.

The GaAs substrates are loaded into an Aixtron AIX 200/4 MOCVD system through a nitrogen glovebox, and heated up to $375{ }^{\circ} \mathrm{C}$ under a TBAs overpressure. Indium droplets are deposited by decomposing TMIn at $375{ }^{\circ} \mathrm{C}$ and 800 mbar for 15 seconds. InP nanowires are grown with precursor flow rates of TMIn $=12.168 \mathrm{~mol} / \mathrm{min}$ and $\mathrm{TBP}=348.592 \mathrm{~mol} / \mathrm{min}$ for 16 minutes. No active dopant source is flowed, and the system pressure is maintained at 100 mbar. Once growth is finished, the system is cooled down under a TBAs overpressure. 


\section{Solid-source CVD growth of InN nanowires}

The growth substrates are single crystal (100) boron-doped silicon with a resistivity of 0.001 - $0.005 \Omega$-cm (MTI Corp.). The substrates are cleaved into $1 \mathrm{~cm}$ by $1 \mathrm{~cm}$ pieces with a diamond scribe and wafer cleaving pliers and rinsed sequentially with acetone, methanol, and isopropanol to remove any powder residue on the surface. The silicon substrates are dipped in a $5 \% \mathrm{HF}$ solution to remove native $\mathrm{SiO}_{2}$. For growths involving gold-catalyzed nanowires, poly-L-lysine solution $(0.1 \% \mathrm{w} / \mathrm{v}$, Ted Pella $)$ is pipetted onto the surface of the substrates for 2 minutes to form a positively charged surface, and then blown off with a nitrogen stream. A colloidal solution of gold nanoparticles (50 nm diameter, BBI Solutions) is then drop cast onto the substrates, with the negatively charged nanoparticles adhering to the surface through electrostatic attraction with the positively charged poly-L-lysine. After 5 minutes, the colloidal solution is blown off with a nitrogen stream.

The silicon substrates are loaded into a single-zone tube furnace with a metal indium source upstream. Both the substrates and indium are in quartz dishes. For the gold-catalyzed InN nanowire growth, $0.8 \mathrm{~g}$ of indium (99.99\%, Alfa Aesar) is placed in the source dish, and the silicon substrates with gold nanoparticles are in a substrate dish $3 \mathrm{~cm}$ downstream within the same heating zone. The furnace is pumped down to a base pressure of 30 mTorr for 15 minutes to remove any trapped oxygen and water vapors, and refilled with argon gas. The argon is pumped out and refilled for three cycles to ensure inert environmental conditions for growth. The furnace is heated up to $550{ }^{\circ} \mathrm{C}$ under a static argon pressure of 35 Torr. Once the temperature setpoint is reached, the argon is emptied and a $80 \% / 20 \%$ mixture of $\mathrm{N}_{2} / \mathrm{NH}_{3}$ is opened at a volumetric rate of $929.9 \mathrm{sccm}$ and a dynamic pressure of 6 Torr. This reduced pressure is used to minimize indium-ammonia reactions in the vapor phase and to promote indium vapor transfer to the substrate surface. The growth is carried out for 240

minutes, after which the gas is switched back to argon at a volumetric flow rate of $70 \mathrm{sccm}$ and a dynamic pressure of 10 Torr. Under these conditions, the furnace is cooled down to $250{ }^{\circ} \mathrm{C}$ and the samples are removed.

For the indium-catalyzed InN nanowire growth, $0.8 \mathrm{~g}$ of indium is placed in the source dish, and the bare silicon substrates are placed in a substrate dish downstream. The temperature gradient of the furnace is used to decouple the indium metal volatilization heating and the growth temperature on the substrate. The furnace is pumped down to a base pressure of 30 mTorr for 15 minutes to remove any trapped oxygen and water vapors, and refilled with argon gas. The argon is pumped out and refilled for three cycles to ensure inert environmental conditions for growth. The furnace is heated up to a nominal value of $700{ }^{\circ} \mathrm{C}$ with argon at a volumetric flow rate of $140 \mathrm{sccm}$ and a dynamic pressure of 3 Torr, with the indium source at 
a measured temperature of $710{ }^{\circ} \mathrm{C}$ and the silicon substrate at a measured temperature of $493{ }^{\circ} \mathrm{C}$. Once the temperature setpoint is reached, these conditions are held for 20 minutes to promote indium transport to the substrate as surface droplets. The gas is switched to a $80 \% / 20 \%$ mixture of $\mathrm{N}_{2} / \mathrm{NH}_{3}$ at a volumetric flow rate of $929.9 \mathrm{sccm}$ and a dynamic pressure of 5 Torr, and the nominal furnace temperature is decreased to $618{ }^{\circ} \mathrm{C}$ with the indium source at a measured temperature of $620{ }^{\circ} \mathrm{C}$ and the silicon substrate at a measured temperature of $410{ }^{\circ} \mathrm{C}$. A reduced pressure is used to minimize indium-ammonia reactions in the vapor phase and to promote indium vapor transfer to the substrate surface. The growth is carried out for 150 minutes, after which the gas is switched back to argon at a volumetric flow rate of $70 \mathrm{sccm}$ and a dynamic pressure of 10 Torr. Under these conditions, the furnace is cooled down to $250{ }^{\circ} \mathrm{C}$ and the samples are removed.

\section{Electrode fabrication and chemicals preparation}

The sample pieces are flipped upside down on a half-cylindrical dish to minimize surface contact. A scratch is made on the backside using a diamond scribe to expose an unoxidized surface, and an ohmic rear contact is made. For silicon, an indium-gallium eutectic is applied, and a copper wire is soldered onto the liquid contact with pure indium.

For InP photocurrent measurements, the non-aqueous electrolyte is prepared using anhydrous methanol (99.8\%, Sigma-Aldrich). The supporting electrolyte salt is $0.1 \mathrm{M} \mathrm{NH}_{4} \mathrm{Cl}$ (99.5\%, Sigma-Aldrich). The redox salt is methyl viologen dichloride (98\%, Sigma-Aldrich), added at a concentration of $40 \mathrm{mM}$. The reference electrode is an $\mathrm{Ag}$ wire, anodized in $\mathrm{HCl}$ to create an $\mathrm{AgCl}$ coating, immersed in a $0.3 \mathrm{M} \mathrm{NH}_{4} \mathrm{Cl}$ filling solution, and encased in a glass tube with a fritted tip (CH Instruments). It is calibrated to the $\mathrm{Fc} / \mathrm{Fc}^{+}$potential using a glassy carbon disk as a working electrode while adding ferrocene (98\%, Sigma-Aldrich) and ferrocenium tetrafluoroborate (technical grade, Sigma-Aldrich) to the solution as redox salts. A $1 \mathrm{~V} /$ sec cyclic voltammetry curve is run around $\mathrm{V}_{o c}$, and the $\mathrm{Fc} / \mathrm{Fc}^{+}$reference is set by taking the half-wave potential between the oxidation and reduction peaks.

For InN photovoltage measurements, the non-aqueous electrolyte is prepared using anhydrous acetonitrile (99.8\%, Sigma-Aldrich). The redox salt is bis(cyclopentadienyl)cobalt(II) (Sigma-Aldrich), added at a concentration of $2 \mathrm{mM}$. The supporting electrolyte salt is 0.1 M TMAHFP ( $\geq 98 \%$, Sigma-Aldrich). The reference electrode is an Ag wire immersed in a $0.1 \mathrm{M}$ TMAHFP/10 $\mathrm{mM} \mathrm{AgNO}_{3}$ filling solution, and encased in a glass tube with a fritted tip ( $\mathrm{CH}$ Instruments). It is calibrated to the $\mathrm{Fc} / \mathrm{Fc}^{+}$potential using a glassy carbon disk as a working electrode while adding ferrocene (98\%, Sigma-Aldrich) and ferrocenium tetrafluoroborate (technical grade, Sigma-Aldrich) to the solution as redox salts. A $1 \mathrm{~V} / \mathrm{sec}$ 
cyclic voltammetry curve is run around $\mathrm{V}_{o c}$, and the $\mathrm{Fc} / \mathrm{Fc}^{+}$reference is set by taking the half-wave potential between the oxidation and reduction peaks. A series of cyclic voltammetry scans are taken around the open-circuit voltage for the solution with the dissolved (cyclopentadienyl)cobalt(II) salt to ensure the presence of both $\mathrm{CoCp}_{2}{ }^{0}$ and $\mathrm{CoCp}_{2}{ }^{+}$species during photovoltage measurements.

The counter electrode for all measurements is a glassy carbon disk integrated within a Kel-F rod (CH Instruments).

\section{Extraction of capacitance from EIS measurements}

The data from EIS measurements is obtained as an impedance with a real component $\left(Z_{r e}\right)$ and an imaginary component $\left(Z_{i m}\right)$ as a function of frequency and applied bias. The impedance is modeled with a four-element circuit shown in Figure S3. $R_{\Omega}$ represents a series resistance that accounts for external resistive losses within the electrochemical circuit, such as electrode wire resistance and supporting electrolyte conductivity. $R_{c t}$ represents a resistance of charge transfer between electrolyte ions and electrons in the semiconductor electrode. $C_{d}$, which is alternatively expressed as $C$ in this work, represents the capacitance at the interface. At an ideal conductor/electrolyte interface, this capacitance arises from adsorbed ion components forming an electric double layer (described as a compact, Helmholtz, or Stern layer). Within a semiconductor/electrolyte interface, however, there is an additional component from depletion within the semiconductor. This is usually the dominant capacitive contribution. $W$ represents a diffusion coefficient representing the regime where the electrochemical reaction at the interface is no longer kinetically limited, but ion transport/mass transfer limited. This is usually the case when the potential bias is well beyond the standard redox potential for the reaction of interest.

The total impedance $\left(Z_{r e}+Z_{i m}\right)$ from the model is obtained by combining $R_{\Omega}, R_{c t}, C_{d}$, and $W$, and comparing the result to the measured impedance data within a Nyquist plot. Through an iterative simplex algorithm in EC-Lab Zfit, the four values are adjusted to best fit the measured data within reason (i.e., the results make sense and are interpretable). This method is applied at different potential biases in order to view the change in capacitance, and thus the semiconductor electrostatics, with applied bias to gain insight into the interface electrostatics. 
(a)

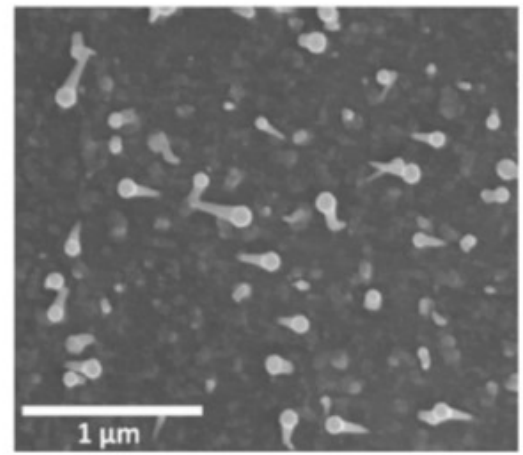

(b)

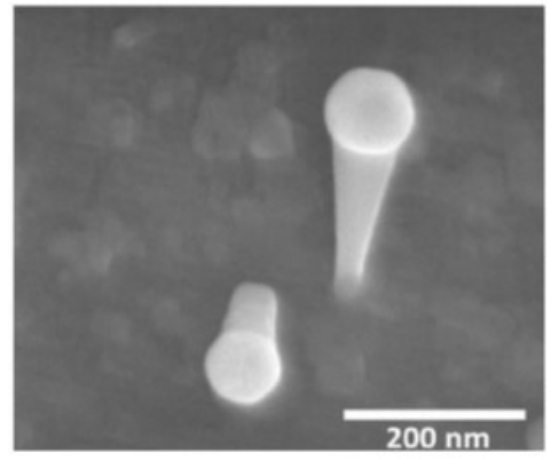

Figure S1: (a) SEM image of InP nanowires grown on (100) GaAs. (b) SEM image of InP nanowires grown on (100) GaAs, under high magnification.

(a)

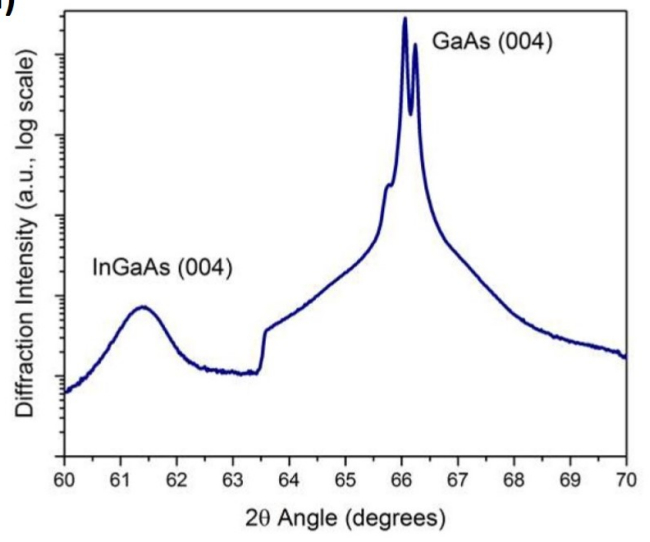

(b)

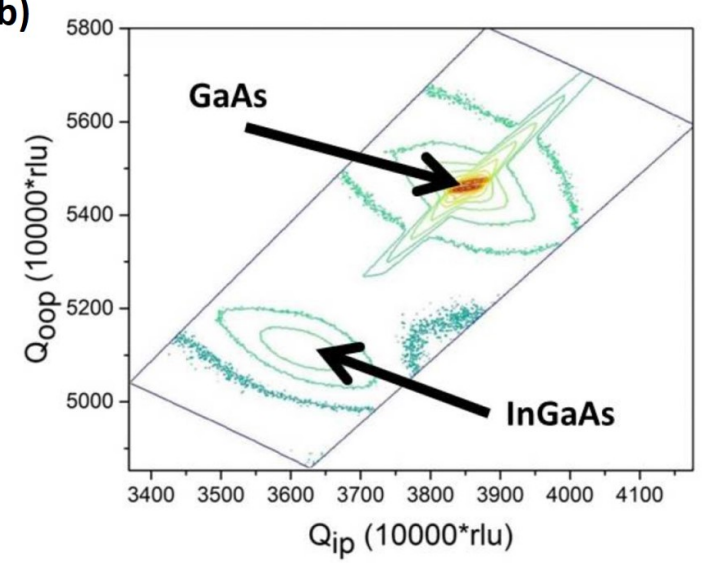

Figure S2: X-ray diffraction scans of InP nanowires grown on GaAs: (a) symmetric, and (b) reciprocal space map, showing that an indium-rich relaxed InGaAs layer is formed at the interface between the wires and substrate. 


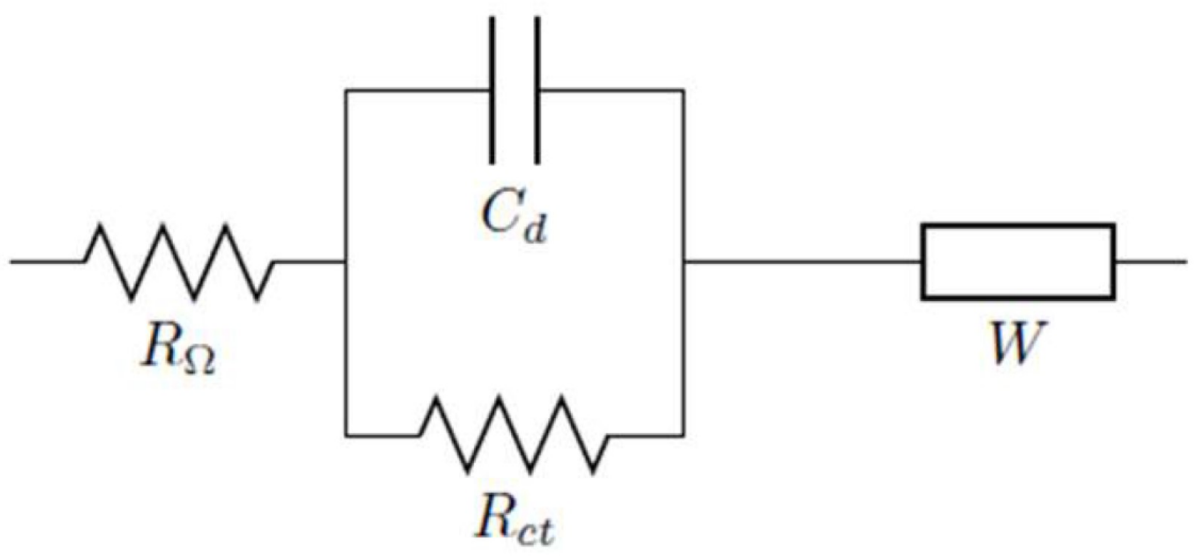

Figure S3: Circuit model used for EIS data analysis. 